\title{
Anisotropic Stresses in Homogeneous Cosmologies
}

\author{
M. A. H. MacCallum and J. M. Stewart \\ Department of Applied Mathematics and Theoretical Physics, Silver Street, Cambridge \\ B. G. SCHMIDT \\ 1. Institut für Theoretische Physik, Hamburg
}

Received March 20, 1970

\begin{abstract}
Recently a certain class of homogeneous world models filled with perfect fluid have been discussed, $[1,2]$. The corresponding results when anisotropic stresses are included are now examined.
\end{abstract}

This paper discusses general-relativistic spacetimes admitting a 3-parameter group of motions acting simply-transitively on spacelike surfaces of homogeneity such that the tangent vectors of the normal congruence are Ricci eigenvectors. In previous papers $[1,2]$ the normals $u^{a}$ were further assumed to be the fluid flow vectors for a perfect fluid, and the aim of this paper is to investigate the validity of previous results when this restriction is relaxed. Physically this will allow us to consider a fluid with 4-velocity $u^{a}$ and anisotropic stresses, but with no net energy flux relative to the fluid flow.

The calculation techniques and group classification follow [1]. The results of [1], Section 2, remain valid, and in particular, $\omega=\dot{u}=0$ and $\partial_{\alpha}$ applied to any covariantly defined quantity gives zero. The energymomentum tensor has the form [9],

$$
T^{a b}=\mu u^{a} u^{b}+p h^{a b}+\pi^{a b}
$$

where $\pi^{a b}=\pi^{(a b)}, \pi^{a}{ }_{a}=\pi^{a b} u_{b}=0 . T^{a b}$ must have the form (1) because $u^{a}$ is assumed to be a Ricci eigenvector. If we interpret $u^{a}$ as the mean fluid flow vector then $\mu$ is the energy density, $p$ the pressure, and $\pi_{a b}$ the anisotropic stress measured in the rest frame of $u^{a}$. The contracted Bianchi identities $T_{; b}^{a b}=0$ read,

$$
\begin{gathered}
\dot{\mu}+\mu \theta+\left(p h^{a b}+\pi^{a b}\right)\left(\frac{1}{3} \theta h_{a b}+\sigma_{a b}\right)=0, \\
3 a^{v} \pi_{v \alpha}+\pi^{\gamma \mu} \varepsilon_{\gamma \alpha \tau} n_{\mu}{ }^{\tau}=0 .
\end{gathered}
$$


(The form of (3) arises from the fact that $\dot{u}_{\alpha}=\partial_{\alpha} p=\partial_{\gamma} \pi_{\alpha}{ }^{\gamma}=0$ for the reason given above.) The only alteration to the field equations is the addition of a term $\pi^{\beta \delta}$ to the right hand side of Eq. (3.4) in [1], and equations derived from it. First we discuss the modifications of the results of [1], without making any physical restriction on $T^{a b}$, i.e. from a purely geometrical standpoint.

Lemma $1(4.1)^{1}$. In all the spacetimes of Class $A$ except those of Bianchi types I and II, there exists an orthonormal tetrad $\left\{\boldsymbol{e}_{a}\right\}$ with $\boldsymbol{u}=\boldsymbol{e}_{0}$ and such that the vectors $\left\{\boldsymbol{e}^{\beta}\right\}$ are Fermi-propagated shear and stress eigenvectors of the flow congruence, and Ricci eigenvectors of the surfaces of homogeneity. The Weyl tensor is then type I or its specialisations $D$ or $O$.

In Bianchi types I and II such a tetrad need not exist. However in these cases the tetrad can be chosen either as shear eigenvectors or as stress eigenvectors or to be Fermi-propagated, and if there is a tetrad having any two of these properties then it has the third.

Lemma 2 (4.2). In all spacetimes of Class A except Bianchi types I and II there exist coordinates $x^{i}$ such that the metric tensor takes the form of [1], (4.8).

These results follow since the proofs of [1] hold except where the $(\alpha \beta)$ field equations $(\alpha \neq \beta)$ are required.

Lemma 3(5.1). The only Class B spacetime in which $a^{\beta}$ is not necessarily a Fermi-propagated shear and stress eigenvector is that with a group of type $V I_{h}$ with $h=-1 / 9$.

The information regarding stress follows from (3).

Lemma 4 (5.2). A spacetime of Class $B$ has $n_{2}=n_{3}$ on an open neighbourhood if and only if there is a group of type $V$.

The proof follows [1] except where it is asserted that $\partial_{0} \sigma_{23}=0$ $\Rightarrow \sigma_{12} \sigma_{13}=0$. However if $n_{2}=n_{3} \neq 0$, [1] (3.3) implies $\sigma_{12}=\sigma_{13}=0$ and so Lemma 4 follows.

We can no longer assert that when there is a group of type $\mathrm{V} \sigma_{\alpha \beta}=0$ $(\alpha \neq \beta)$. The argument concerning the case $\sigma_{23}=0$ similarly fails and so do the proofs of Lemma 5.3 and Theorems 6.1 and 6.2.

If in Class $\mathrm{A}$ the eigenvectors of $n_{\alpha \beta}, \sigma_{\alpha \beta}$ and $\pi_{\alpha \beta}$ coincide and are thus Fermi-propagated, we have the metric form [1] (4.8). For the $n_{\alpha}^{\alpha}=0$ cases except Bbii we can similarly obtain the metric form [1] (6.6), provided Theorem 6.1 holds, and that in type $\mathrm{V} \sigma_{23}=\pi_{23}=0$. When these metric forms are available we can write the stresses as derived from

\footnotetext{
1 The numbering in brackets refers to the corresponding result in [1].
} 
a potential (cf. [3]). We have,

$$
\begin{aligned}
& X \equiv l_{1} \equiv e^{-\Omega+\beta_{1}}, \quad Y \equiv l_{2} \equiv e^{-\Omega-\frac{\beta_{1}}{2}+\frac{\sqrt{3} \beta_{2}}{2}}, \quad Z \equiv l_{3} \equiv e^{-\Omega-\frac{\beta_{1}}{2}-\frac{\sqrt{3} \beta_{2}}{2}}, \\
& \theta_{1}=\left(l_{1}\right)^{\bullet} / l_{1}, \theta_{2}=\left(l_{2}\right)^{\bullet} / l_{2}, \theta_{3}=\left(l_{3}\right)^{*} / l_{3} \text {, and } \pi_{\alpha \beta}=\operatorname{diag}\left(\pi_{1}, \pi_{2}, \pi_{3}\right) \text {. }
\end{aligned}
$$

Substituting in (2) we obtain

$$
0=d\left(\mu l^{3}\right)+\sum_{v}\left(p+\pi_{v}\right) \theta_{v} l^{3} d t=d\left(\mu l^{3}\right)+\sum_{v} \frac{\left(p+\pi_{v}\right) l^{3} d\left(l_{v}\right)^{2}}{2 l_{v}{ }^{2}}
$$

where $l^{3} \equiv l_{1} l_{2} l_{3} \equiv e^{-3 \Omega}$. Thus in a frame of invariant reciprocal vectors with $l_{v}^{-2}=g^{v v}$,

$$
\sqrt{-g} T_{\lambda \nu}= \begin{cases}0 & (\lambda \neq v) \\ 2 \frac{\partial(\mu \sqrt{-g})}{\partial\left(l_{v}{ }^{-2}\right)} & (\lambda=v) .\end{cases}
$$

In the $\left(\Omega \beta_{1} \beta_{2}\right)$ parametrisation introduced by Misner and used by others, $[5-8]$, we may express the Einstein equations in the following form,

$$
\left.\begin{array}{rl}
4 \Omega^{\bullet 2} & =\frac{4}{3}(\mu+\Lambda)+\left(\beta_{1}^{\cdot 2}+\beta_{2}^{\cdot 2}\right)-\frac{2 R^{*}}{3} \\
\left(e^{-3 \Omega} \beta_{1}^{\bullet}\right)^{\bullet} & =-\frac{e^{-\Omega}}{2} \frac{\partial V_{1}}{\partial \beta_{1}}-\frac{1}{2} \frac{\partial V_{2}}{\partial \beta_{1}} \\
\left(e^{-3 \Omega} \beta_{2}^{\bullet}\right)^{\bullet} & =-\frac{e^{-\Omega}}{2} \frac{\partial V_{1}}{\partial \beta_{2}}-\frac{1}{2} \frac{\partial V_{2}}{\partial \beta_{2}} \\
\dot{\mu} & =3 \Omega^{\bullet}(\mu+p)+\frac{3}{4} \beta_{i} \frac{\partial V_{2}}{\partial \beta_{1}}+\frac{3}{4} \beta_{2} \cdot \frac{\partial V_{2}}{\partial \beta_{2}}
\end{array}\right\}
$$

where $V_{1}=-\frac{2}{3} R^{*} e^{-2 \Omega}, V_{2}=4 \mu / 3, R^{*}$ being the curvature of the surfaces of transitivity. The form (6) is of course not very useful unless some physical assumptions allowing us to calculate $V_{2}$ are made. One could now proceed by writing in the notation of [8],

$$
\Phi=\left(\frac{4}{3} \mu+\beta_{1}^{\cdot 2}+\beta_{2}^{\cdot 2}-\frac{2 R^{*}}{3}\right) e^{-6 \Omega}
$$

( $\Phi$ is Misner's $\Lambda$, cf. [6]), and an analysis similar to [6] and [8] could be developed, but this will not be studied here. 
We now discuss possible prescriptions for the shearing stress. If microscopic collisions occur and the relativistic Boltzmann equation can be applied, an initial value problem can be formulated. This could be used to describe the early stages in the Universe at temperatures above $10^{10}{ }^{\circ} \mathrm{K}$. (See also [11].) We shall assume that $u^{a}$ is parallel to the total rest mass flux vector of the particles. Apart from a very short period immediately after initial conditions have been imposed, the Boltzmann equation will have a "normal solution", that is the distribution function depends only on the rest mass density $\varrho$, the mean 4-velocity $u^{a}$, the temperature $T$, and their first derivatives, [3]. The homogeneity of our models implies that the stress tensor $\pi_{a b}$ can be written as a function of the shear tensor $\sigma_{a b}$, and in fact we find from kinetic theory, [3], that,

$$
\pi^{a b}=-\lambda \sigma^{a b}, \lambda \text { a scalar } .
$$

Clearly from (6), $\pi_{a b}$ and $\sigma_{a b}$ can be simultaneously diagonalised except for a very short period immediately after the imposition of initial conditions, and therefore [1] Theorems 4.1 and 4.2 hold in their original form, so that Bianchi types I, II are no longer exceptional. The arguments concerning type $\mathrm{V}$ and the case $\sigma_{23}=0$ also hold. If $\pi_{a b}$ has the form (6) Lemma 6.1 and Theorem 6.2 hold in their original form. When a normal solution of the Boltzmann equation is applicable the heat flow vector $q^{a}$ will take the form $[3,9]$

$$
q^{a}=-\eta h^{a b}\left(T_{, b}+T \dot{u}_{b}\right)
$$

where $T$ is the temperature and $\eta$ is a scalar. The homogeneity of our models implies that $q^{a}=0$. A similar argument applies to diffusion flows in the case of a multicomponent fluid, and these two results justify our choice of the energy-momentum tensor (1).

If collisions do not occur, the above remarks do not apply. It is no longer possible to apply normal solution theory, and the stress tensor depends not only on the current values of the fluid variables but also on the Cauchy data for the initial value problem. This type of behaviour would occur for example with collision-free neutrinos (photons) after the temperatures of the radiation in the Universe had dropped below $10^{10}{ }^{\circ} \mathrm{K}\left(10^{4}{ }^{\circ} \mathrm{K}\right)$ respectively, (see e.g. $\left.[3,5,10]\right)$. Misner has shown that (4), (5) hold for homogeneous collision-free radiation in Bianchi type I, [5] and Stewart [10] has given an explicit integration for locally rotationally symmetric Bianchi type I models. These authors have suggested that (6) can be expected to hold in cases where the shear anisotropy is small. However it is difficult to find solutions in general because there are an insufficient number of homogeneous constants of the motion to characterise all of the homogeneous distribution functions [4]. 


\section{References}

1. Ellis, G. F. R., MacCallum, M. A. H.: Commun. Math. Phys. 12, 108 (1969).

2. Schmidt, B. G.: Commun. Math. Phys. 15, 329 (1969).

3. Stewart, J. M.: Ph.D. Thesis, University of Cambridge (1969).

4. MacCallum, M. A. H., Ellis, G. F. R.: A class of homogeneous cosmological models II: Observations, Commun. Math. Phys. to appear (1970).

5. Misner, C. W.: Astrophys. J. 151, 431 (1968).

6. - Phys. Rev. Letters 22, 1071 (1969).

7. Hawking, S. W.: Month. Not. Roy. Astron. Soc. 142, 129 (1969).

8. MacCallum, M. A. H.: A class of homogeneous cosmological models III : Asymptotic behaviour. Shortly to be submitted for publication (1969).

9. Ehlers, J.: Akad. Wiss. Litt. (Mainz), Abhandl. Math. Nat. K1. No. 11 (1961).

10. Stewart, J. M.: Astrophys. Letters 2, 33 (1968).

11. - Month. Not. Roy. Astron. Soc. 145, 347 (1969).

M. A. H. MacCallum Department of Applied Mathematics and Theoretical Physics

Silver Street

Cambridge, England 
